\title{
THEOREMS ON THE LOGARITHMIC POTENTIAL
}

\author{
By T. J. I'A. Bromwich.
}

[Read March 9th, 1905.-Received March 16th, 1905.]

THE following investigation contains, in $\S 2$, conditions, necessary and sufficient, for the existence of the second derivates of the logarithmic potential produced by a surface distribution at points within the area which carries the surface density. The surface density is assumed to be generally continuous, the discontinuities occurring along regular curves, but no assumption is made as to the existence of first derivates of the density. As a preliminary, an investigation is given, in $\$ 1$, of the first derivates of the potential ; this contains no essential novelty, although it does not appear in most of the ordinary text-books.

The corresponding problem for the first derivates of the logarithmic potential of a line distribution, at a point on the curve carrying the density, is discussed in $\S 4$, and these are compared, in $\$ 5$, with the limiting values of the derivates at a point which is made to epproach the curve along a path (not touching the curve). For the work in these paragraphs we need a lemma of the integral calculus, given in $\$ 3$.

The mode of investigation used in $\S \S 2,4,5$ is suggested by the papers of Petrini* on the corresponding problems of the Newtonian potential, but many changes in details have been made. Although, as may be anticipated, there is considerable similarity between Petrini's results and those given below, yet there are some unexpected points of difference.

In $\S 6$ the results are compared with those of previous writers, and in $\S 7$ an indication is given as to certain simplifications possible when the density is continuous. $t$

\section{The First Derivates of the Logarithmic Potential of a Surface Distribution.}

If $V$ is the logarithmic potential at $(x, y)$ of a surface distribution $\rho$ over a plane area $S$, we have

$$
V=-2 \gamma \iint \rho \log r d \xi d \eta
$$

- Öfversigt af $K$. Vet. Akaul. Förh. Stockholm, Bd. crir., 1900, pp. 225 and 867.

$\dagger \oint \oint 6$ and 7 have been added to the paper as originally written.-April 30th, 1905.

$\mp$ The constants are chosen so that $\rho$ may represent the volume density of the equivalent cylindrical distribution, attracting according to the Newtonian law, $\gamma$ being the gravitation constant. 
where $r^{2}=(x-\xi)^{2}+(y-\eta)^{2}$, and the integral is to be extended over the whole of the area $S$. Thus, if $V^{\prime}$ is the potential at $(x+h, y)$,

$$
V^{\prime}=-2 \gamma \iint \rho \log r^{\prime} d \hat{\xi} d \eta,
$$

where $r^{\prime 2}=(x+h-\xi)^{2}+(y-\eta)^{2}$.

Then, if $(x, y)$ is external to the area $S$, there is no difficulty in seeing that the equation

$$
\lim _{h=0}\left(V^{\prime}-V\right) / h=2 \gamma \iint\left[\rho(\xi-x) / r^{2}\right] d \xi d \eta
$$

is correct; in fact, $\left(\log r^{\prime}-\log r\right) / h$ converges to the value $(x-\xi) / r^{2}$ as $h$ tends to zero, and the convergence is easily seen to be uniform for all points $(\xi, \eta)$ of the area $S$.

But the truth of the equation is not quite so readily established in case $(x, y)$ falls within the area $S$; for then the convergence of the above expression to its limit is no longer uniform throughout the area.

To deal with this case we proceed on the lines of the proof given by Bouquet for the corresponding three-dimensional case. * Plainly, if the theorem can be established for a small circular area, with its centre at $(x, y)$, it will be true in general; for the preceding result can be applied to the portion of the area which falls outside the circle.

$$
\text { Now } \quad \frac{r-V}{h}=2 \gamma \iint \rho\left[\frac{1}{h} \log \frac{r}{r^{\prime}}\right] d \xi d \eta \text {, }
$$

and denote by $X$ the integral

$$
2 \gamma \iint \rho \frac{\xi-x}{r^{2}} d \xi d \eta
$$

both being extended over the circular area. It is easily seen that the integral $X$ is convergent (and, indeed, absolutely convergent) by expressing the integral in polar coordinates $r, \theta$. Now, since $\left|r^{\prime}-r\right| \leqslant|h|$, it follows that +

$$
\left|\log \frac{r}{r^{\prime}}\right|<\frac{1}{2}\left|\frac{r}{r^{\prime}}-\frac{r^{\prime}}{r}\right|<\frac{1}{2}|h|\left(\frac{1}{r}+\frac{1}{r^{\prime}}\right) .
$$

Hence

$$
\left|\frac{V^{\prime}-V}{h}\right|<\gamma \iint|\rho|\left(\frac{1}{r}+\frac{1}{r^{\prime}}\right) d \xi d \eta \text {. }
$$

Now $\iint(|\rho| / r) d \xi d \eta \leqslant 2 \pi a M$, if $a$ is the radius of the circle and $M$ is the upper limit of $|\rho|$ (supposed finite) within the circle. Also $\iint\left(|\rho| / r^{\prime}\right) d \xi d \eta$ is less than $\iint\left(\mid r / r^{\prime}\right) d \xi d \eta$ extended over a circle of radius

- See, for instance, Picard's Traite $l^{\prime}$ Analyse (t. 1., p. 161); the earliest place in which the proof occurs seems to be the first edition of Briot's Thiorie Micanique de la Chaleur (p. 194). real.

+ Fur, if $p=\log q$, then $q-1 / q=2 \sinh p$, or $|q-1 / q|>2|p|$, the numbers being supposed 
$a+|h|$ with centre at $(x+h, y)$; and this gives

$$
\iint\left(|\rho| \gamma r^{\prime}\right) d \xi d \eta<2 \pi(a+|h|) M<4 \pi a M, \text { if }|h|<a ;
$$

so that

$$
\left|\left(V^{\prime}-V\right) h^{-1}\right|<6 \pi y a M \text {. }
$$

Next consider the integral $X$; we have

$$
|X|<2 \gamma \iint(|\rho| / r) d \xi d \eta
$$

or

$$
|X|<4 \pi \gamma a M \text {. }
$$

By combining these two results we find

$$
\left|h^{-1}\left(V^{\prime}-V\right)-X\right|<1 \dot{0}_{\pi \gamma a M}
$$

If, therefore, $\epsilon$ is any assigned small positive number, let $a$ be chosen so that $a \leqslant \frac{1}{10} \epsilon / \pi \gamma M$; and this can be done, because $M$, or at least a number greater than $M$, can be fixed beforehand.

Then $\left|h^{-1}\left(V^{\prime}-V\right)-X\right|<\epsilon$, if $|h|<a$, and consequently

$$
\lim _{h=0}\left(V^{\prime}-V\right) / h=X,
$$

which is the desired result.*

It may be noticed incidentally that the condition, used above, that $|\rho|$ is to have a finite upper limit in the part of the area $S$ which is in the neighbourhood of the point $(x, y)$ is by no means necessary, and it is easy to see that the final conclusion is correct provided that the two integrals

are convergent.

$$
\iint(\rho / r) d \xi d \eta, \quad \iint\left(\rho / r^{\prime}\right) d \xi d \eta
$$

\section{The Second Derivates of the Logarithmic Potential of a Surface}

\section{Distribution.}

If the density $\rho$ is always less in absolute value than a fixed number, we have seen $(\$ 1)$ that the first derivates of the potential are given by the integrals

$$
\frac{\partial V}{\partial x}=2 \gamma \iint \rho \frac{\xi-x}{r^{2}} d \xi d \eta, \quad \frac{\partial V}{\partial y}=2 \gamma \iint \rho \frac{\eta-y}{\eta^{2}} d \xi d \eta,
$$

and that these results are correct, whether $(x, y)$ is internal or external to the distribution.

Thus, to find $\partial^{2} V / \partial x^{2}$, we have to consider the limit (should it exist)

$$
\lim _{h=0} \frac{2 \gamma}{h} \iint \rho\left[\frac{\xi-x-h}{r^{\prime 2}}-\frac{\xi-x}{r^{2}}\right] d \xi d \eta,
$$

where $r^{2}=(\xi-x-h)^{2}+(\eta-y)^{2}$.

- Harnack (Theorie des logarithmischen Potentiales, Kap. 1, § 5) has obtained this result, using the method of integrating $X$, instead of differentiating $V$. 
If $(x, y)$ is not an internal point, there is no difficulty in proving (by the aid of uniform convergence or otherwise) that the second derivate is obtained by differentiating under the integral sign, so that

$$
\frac{\partial^{2} V}{\partial x^{2}}=2 \gamma \iint \rho \frac{(\xi-x)^{2}-(\eta-y)^{2}}{r^{4}} d \xi d \eta,
$$

with similar values for the other second derivates.

But the discussion is entirely different if $(x, y)$ is an internal point, and it will be necessary to postulate some further restriction on $\rho$, in addition to the condition that $|\rho|$ is to have a finite upper limit. In order to discuss the second derivates at the edge of the distribution, we shall not restrict $\rho$ to be continuous in the neighbourhood of $(x, y)$, but it will be assumed that $\rho$ is continuous on each radius through $(x, y)$. Thus, if we write

$$
\hat{\xi}-x=r \cos \theta, \quad \eta-y_{i}=r \sin \theta,
$$

we shall assume that the limit $\lim _{r=0} \rho$ gives a definite function of $\theta$; and if $\rho$ happens to be completely continuous, the function of $\theta$ will reduce to a constant, in which case the method of $\$ 7$ can be used.

We may restrict the distribution to lie within a circle, with centre $(x, y)$, of an arbitrarily small radius $a$; for the second derivates of the potential due to the distribution outside the circle can be calculated by differentiating under the integral sign. Now the integral to be considered is absolutely convergent, and may therefore be written in the form

$$
\frac{2 \gamma}{h} \int_{0}^{2 \pi} d \theta \int_{0}^{a} \rho\left[\frac{r^{2} \cos \theta-h r}{r^{2}-2 h r \cos \theta+h^{2}}-\cos \theta\right] d r
$$

For the sake of definiteness, suppose $h$ to be positive and less than $a$; the necessary modifications when $h$ is negative will be recognized without difficulty. Then write $r=h t$, and the integral becomes

$$
2 \gamma \int_{0}^{2 \pi} d \theta \int_{0}^{a / h} \rho\left(\frac{t^{2} \cos \theta-t}{t^{2}-2 t \cos \theta+1}-\cos \theta\right) d t,
$$

where it is to be remembered that $\rho$ is a function of $h t$ and $\theta$. But this is the same as $A+B+C$, where

$$
\begin{aligned}
& A=2 \gamma \int_{0}^{2 \pi} d \theta \int_{0}^{1} \rho\left(\frac{t^{2} \cos \theta-t}{t^{2}-2 t \cos \theta+1}-\cos \theta\right) d t, \\
& B=2 \gamma \int_{0}^{2 \pi} d \theta \int_{1}^{a / h} \rho\left(\frac{t^{2} \cos \theta-t}{t^{2}-2 t \cos \theta+1}-\cos \theta-\frac{\cos 2 \theta}{t}\right) d t, \\
& C=2 \gamma \int_{0}^{2 \pi} \cos 2 \theta d \theta \int_{1}^{a / h}(\rho / t) d t=2 \gamma \int_{0}^{2 \pi} \cos 2 \theta d \theta \int_{h}^{a}(\rho / \gamma) d r .
\end{aligned}
$$


Write now $f(\theta)=\lim _{r=0} \rho$, and then there is no difficulty in seeing that

$$
\lim _{h=0} A=2 \gamma \int_{0}^{2 \pi} f(\theta) d \theta \int_{0}^{1}\left(\frac{t^{2} \cos \theta-t}{t^{2}-2 t \cos \theta+1}-\cos \theta\right) d t,
$$

because * $\left|\frac{t^{2} \cos \theta-t}{t^{2}-2 t \cos \theta+1}-\cos \theta\right|<\left(t^{2}-2 t \cos \theta+1\right)^{-b}+1$

and

$$
\int_{0}^{1}\left[\left(t^{2}-2 t \cos \theta+1\right)^{-\frac{1}{2}}+1\right] d t=1+\log \left(1+\operatorname{cosec} \frac{1}{2} \theta\right) .
$$

Now, since the integral $\int_{0}^{2 \pi}\left[1+\log \left(1+\operatorname{cosec} \frac{1}{2} \theta\right)\right] d \theta$ is convergent, it is clear that the value given for $\lim _{h=0} A$ will be certainly correct if the function $\rho$ approaches its limit $f(\theta)$ uniformly for all values of $\theta$ from 0 to $2 \pi$.

To deal with $B$, let the integration with respect to $t$ be taken in two stages, from 1 to 2 and from 2 to $a / h$ (assuming, as we may, that $h<\frac{1}{2} a$ ); then, if for the present we write

$$
F(t, \theta)=\frac{t^{2} \cos \theta-t}{t^{2}-2 t \cos \theta+1}-\cos \theta-\frac{\cos 2 \theta}{t},
$$

we have $\quad \frac{B}{2 \gamma}=\int_{\emptyset}^{2 \pi} d \theta \int_{1}^{2} \rho F(t, \theta) d t+\int_{0}^{2 \pi} d \theta \int_{2}^{a / h} \rho F(t, \theta) d t$.

By an argument exactly similar to that which was used for finding $\lim _{h=0} A$, we see that $\dagger$

$$
\lim _{h=0} \int_{0}^{2 \pi} d \theta \int_{1}^{2} \rho F(t, \theta) d t=\int_{0}^{2 \pi} f(\theta) d \theta \int_{1}^{2} F(t, \theta) d t .
$$

Next, we have the identity

$$
\begin{aligned}
& \int_{0}^{2 \pi} d \theta \int_{2}^{a / h} \rho F(t, \theta) d t-\int_{0}^{2 \pi} f(\theta) d \theta \int_{2}^{\infty} F(t, \theta) d t \\
& =\int_{0}^{2 \pi} d \theta \int_{2}^{N}[\rho-f(\theta)] F(t, \theta) d t+\int_{0}^{2 \pi} d \theta \int_{N}^{a / h} \rho F(t, \theta) d t-\int_{0}^{2 \pi} f(\theta) d \theta \int_{N}^{\infty} F(t, \theta) d t,
\end{aligned}
$$

where $N$ is any number subject to the restriction $N<a / h$. Thus

$$
\begin{aligned}
& \left|\int_{0}^{2 \pi} d \theta \int_{2}^{a / h} \rho F(t, \theta) d t-\int_{0}^{2 \pi} f(\theta) d \theta \int_{2}^{\infty} F(t, \theta) d t\right| \\
& \quad<\int_{0}^{2 \pi} d \theta \int_{2}^{N}|\rho-f(\theta)||F(t, \theta)| d t+M \int_{0}^{2 \pi} d \theta\left[\int_{N}^{a / h}|F(t, \theta)| d t+\int_{N}^{\infty}|F(t, \theta)| d t\right],
\end{aligned}
$$

where $M$ is the upper limit to $|\rho|$ (and therefore to $|f(\theta)|$ also) within the circle $r=a$.

- For $\left|t^{2} \cos \theta-t\right| \leqslant|1-t \cos \theta| \leqslant\left(1-2 t \cos \theta+t^{2}\right)^{1}$.

+ In fact, here

$\left|F^{\prime}(t, \theta)\right|<2\left[1+\left(t^{3}-2 t \cos \theta+1\right)^{-1}\right]$ and $\int_{1}^{2}\left[1+\left(t^{2}-2 t \cos \theta+1\right)^{-1}\right] d t<1+\log \left(3 \operatorname{cosec} \frac{1}{3} \theta\right)$. 
Now

$$
F(t, \theta)=\frac{t \cos 3 \theta-\cos 2 \theta}{t\left(t^{2}-2 t \cos \theta+1\right)}
$$

so that

$$
|F(t, \theta)|<\frac{t+1}{t(t-1)^{2}}
$$

and consequently* $\int_{2}^{N}|F(t, \theta)| d t<\int_{2}^{\infty} \frac{t+1}{t(t-1)^{2}}<2$,

while

$$
\int_{N}^{\omega / h}|F(t, \theta)| d t<\int_{N}^{\infty}|F(t, \theta)| d t<\frac{2}{N-1} .
$$

Let $\epsilon$ be any arbitrarily assigned small positive number; then choose $N$ so that $\quad 8 \pi M /(N-1)<\frac{1}{2} \epsilon$, or $N-1>16 \pi M / \epsilon$.

Next choose $H$ so that

$$
H<a / N \text {, and }|\rho-f(\theta)|<\epsilon / 8 \pi \text {, if } h<H \text {, }
$$

for all values of $\theta$ from 0 to $2 \pi$, and for all values of $t$ from 2 to $N$. Such a choice of $H$ is possible, because the limit of $\rho$ is $f(\theta)$ when the product $h t$ tends to zero, and the convergence of $\rho$ to its limit $f(\theta)$ has been supposed uniform with respect to $\theta$; and $N$ has been fixed above.

It is now clear that

$$
\begin{aligned}
& \begin{aligned}
\mid \int_{0}^{2 \pi} d \theta \int_{2}^{a / h} \rho F(t, \theta) d t & -\int_{0}^{2 \pi} f(\theta) d \theta \int_{2}^{\infty} F(t, \theta) d t \mid \\
& <\int_{0}^{2 \pi} d \theta \int_{2}^{N}(\epsilon / 8 \pi)|F(t, \theta)| d t+2 M \int_{0}^{2 \pi} d \theta \int_{N}^{\infty}|F(t, \theta)| d t \\
& <4 \pi(\epsilon / 8 \pi)+2 M[4 \pi /(N-1)]<\epsilon,
\end{aligned} \\
& \text { provided that } h<H .
\end{aligned}
$$

Hence $\quad \lim _{h=0} \int_{0}^{2 \pi} d \theta \int_{2}^{\alpha / h} \rho F(t, \theta) d t=\int_{0}^{2 \pi} f(\theta) d \theta \int_{2}^{\infty} F(t, \theta) d t$.

If we combine the value found for $\lim _{h=0} A$ with the values obtained from the two parts of $B$, it will be clear that

$$
\begin{aligned}
\lim _{h=0}(A+B)=2 \gamma \int_{0}^{2 \pi} f(\theta) d \theta & {\left[\int_{0}^{1}\left(\frac{t^{2} \cos \theta-t}{t^{2}-2 t \cos \theta+1}-\cos \theta\right) d t\right.} \\
& \left.\quad+\int_{1}^{\infty}\left(\frac{t^{2} \cos \theta-t}{t^{2}-2 t \cos \theta+1}-\cos \theta-\frac{\cos 2 \theta}{t}\right) d t\right] .
\end{aligned}
$$

The integrals with respect to $t$ can be found without difficulty, and they givet

* The indefinite integral is given by $\int \frac{t+1}{t(t-1)^{2}} d t=-\frac{2}{t-1}+\log \frac{t}{t-1}$.

+ If the upper limit of the second integral is $X$ (instead of $\infty$ ), the result is

$$
\frac{1}{2} \cos 2 \theta \log \left(1-\frac{2}{y^{2}} \cos \theta+\frac{1}{N^{-2}}\right)-\sin 2 \theta \arctan \frac{N \sin \theta}{1-N \cos \theta}
$$

where the angle is between 0 and $\pi$ if $\sin \theta$ is positive, between 0 and $-\pi$ if $\sin \theta$ is negative. 


$$
\begin{array}{r}
\int_{0}^{1}\left(\frac{t^{2} \cos \theta-t}{t^{2}-2 t \cos \theta+1}-\cos \theta\right) d t+\int_{1}^{\infty}\left(\frac{t^{2} \cos \theta-t}{t^{2}-2 t \cos \theta+1}-\cos \theta-\frac{\cos 2 \theta}{t}\right) d t \\
=(\theta-\pi) \sin 2 \theta,
\end{array}
$$

where $\theta$ is to be taken between 0 and $2 \pi$. Thus

$$
\lim _{h=0}(A+B)=2 \gamma \int_{0}^{2 \pi}(\theta-\pi) \sin 2 \theta f(\theta) d \theta .
$$

It is now clear that the existence of the second derivate depends on the existence of the limit $\lim _{h=0} C$, and, if this limit exists, we have

$$
\frac{\partial^{2} V}{\partial x^{2}}=2 \gamma \int_{0}^{2 \pi}(\theta-\pi) \sin 2 \theta f(\theta) d \theta+\lim _{h=0} 2 \gamma \int_{0}^{j \pi \pi} \cos 2 \theta d \theta \int_{h}^{a}(\rho / r) d r .
$$

It is to be observed that this second derivate is at present only onesided; that is to say, $h$ has been restricted to be positive. But, as we shall see on p. 352, it is not usually possible to obtain a second derivate which is valid for both signs of $h$, unless $\rho$ is completely continuous, so that $f(\theta)$ is a constant.

The simplest kind of discontinuous density occurs when $\rho$ is constant, say $\rho_{1}$, on one siae of a regular curve passing through $(x, y)$; and a ciiî́erent constant, say $\rho_{2}$, on the other side of the curve.

Suppose that the positive direction of $x$ falls in the $\rho_{1}$ region: we have then only to consider the value of that part * of the integral $C$ which arises from the space included between the curve and iis tangent at $(x, y)$. If we take as the other boundaries two circles of radii $r_{1}, r_{2}$, the corresponding part of $C$ is numerically less than

$$
2 \gamma\left|\rho_{1}-\rho_{2}\right| \int_{r_{1}}^{r_{2}}\left(\phi_{l} r\right) d r \quad\left(r_{2}>r_{1}\right)
$$

where $\phi$ is the angle between the radius $r$ and the

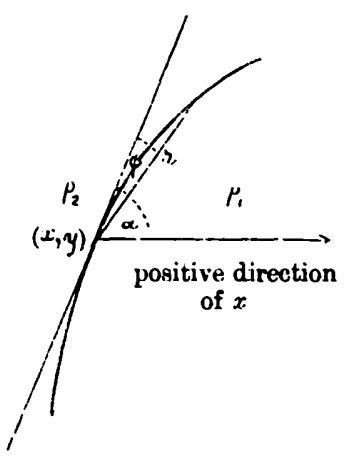
tangent to the curve at $(x, y)$. But $\lim _{r=0}(2 \phi / r)$ is the curvature at $(x, y)$, which we assume to be finite; and consequently the last integral tends to zero with $r_{2}$, whatever $r_{1} / r_{2}$ may be ; thus $C$ has a limit when $h$ tends to zero. Further this limit is less than

$$
2 \gamma\left|\rho_{1}-\rho_{2}\right| \int_{0}^{\prime \prime}(\phi / r) d r
$$

- For the rest of the integral is equal to

$$
2 \gamma(\log a / h)\left[\rho_{1} \int_{0}^{\epsilon} \cos 2 \theta d \theta+\rho_{2} \int_{a}^{+\infty} \cos 2 \theta d \theta+\rho_{1} \int_{\alpha+\infty}^{\pi+} \cos 2 \theta d \theta\right]=0 .
$$


$\phi$ being regarded as always positive; and consequently the limit of $C$ may be made as smail as we please by proper choice of $a$. Thus the derivate $\partial^{2} V / \partial x^{2}$ exists and is given effectively by the integral

$$
\begin{aligned}
2 \gamma \int_{0}^{2 \pi}(\theta-\pi) & \sin 2 \theta f(\theta) d \theta \\
= & 2 \gamma\left[\rho_{1} \int_{0}^{a}(\theta-\pi) \sin 2 \theta d \theta+\rho_{2} \int_{a}^{\pi+a}(\theta-\pi) \sin 2 \theta d \theta\right. \\
& \left.+\rho_{1} \int_{\pi+a}^{2 \pi}(\theta-\pi) \sin 2 \theta d \theta\right] \\
= & -2 \pi \gamma \rho_{1}+\pi \gamma\left(\rho_{1}-\rho_{2}\right) \cos 2 \alpha .
\end{aligned}
$$

From this result it is plain that, as stated on p. 351, the derivate is, in general, one-sided only; for, if the sign of $h$ is changed, we produce the same effect on the derivate as by interchanging $\rho_{1}$ and $\rho_{2}$. The new derivate is

$$
-2 \pi \gamma \rho_{2}+\pi \gamma\left(\rho_{2}-\rho_{1}\right) \cos 2 \alpha,
$$

which is only equal to the former one if $\cos 2 a=1$, or if the axis of $x$ is parallel to the tangent to the curve bounding the regions $\rho_{1}$ and $\rho_{2}$.

However, if the point $(x, y)$ is at an angle of the boundary formed by two regular curves, even the one-sided derivates do not exist; except for two special directions. We note first that the curves may be replaced by their tangents (provided

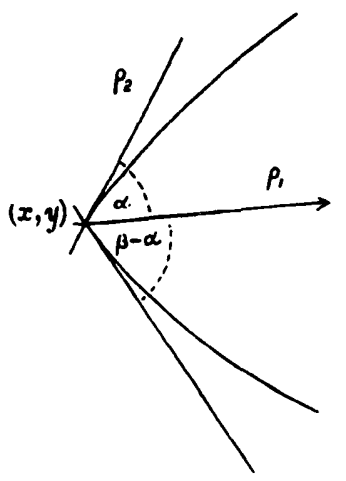
that the curvature of each curve is finite); the argument on this point is the same as above (p. 351), and need not be repeated. Then the integral $C$ becomes

$$
\begin{aligned}
2 \gamma \log (a / h)\left[\rho_{1} \int_{0}^{a} \cos 2 \theta d \theta+\rho_{2}\right. & \left.\int_{\alpha}^{2 \pi+a-\beta} \cos 2 \theta d \theta+\rho_{1} \int_{2 \pi+a-\beta}^{2 \pi} \cos 2 \theta d \theta\right] \\
& =\gamma \log (a / h)\left(\rho_{1}-\rho_{2}\right)[\sin 2 \alpha+\sin (2 \beta-2 \alpha)] \\
& =2 \gamma \log (a / h)\left(\rho_{1}-\rho_{2}\right) \sin \beta \cos (\beta-2 \alpha),
\end{aligned}
$$

where $\beta$ is the angle between the two tangents. Hence, excluding the case $\sin \beta=0$ (which is not essentially different from the case of a single regular curve), we see that $\lim _{h=0} C$ can only exist if $a=\frac{1}{2} \beta \pm \frac{1}{4} \pi$; this gives two special directions, each making an angle of $\frac{1}{4} \pi$ with the bisector of the angle between the two tangents.*

* In the above calculation it was tacitly assumed that $\beta$ was equal to $t \pi$ at least, but it is easily seen, by a fresh integration, that the result is true even if $\beta$ is less than $f \pi$. 
This conclusion can be verified for the special case of a right angle by reference to Maxwell's results * on the "geometrical mean distance" of a point and a rectangle; his formula shews that, at a corner of the rectangle, the second derivates exist only for the two directions which coincide with the sides of the rectangle. Care must be taken, however, to notice that the second derivates of the function obtained from Maxwell's formula are not precisely the same as those given by the results on the last page; for our results refer only to the potential of the area contained within a small circle of radius $a$, whose centre is at the corner; while Maxwell's calculation relates to the whole rectangle. Still, the two formulæ should give the same difference between the derivates taken in opposite directions along the same side of the rectangle; and this appears to be verified, the difference being $2 \pi \gamma\left(\rho_{1}-\rho_{2}\right)$.

The results obtained for the logarithmic potential naturally lead to the conjecture that some analogous properties hold for the Newtonian potential. It appears, by using Petrini's test (l.c.), that, at a conical point on the surface of a solid (of uniform density), the potential has second derivates only for directions which lie on the surface of a quadric cone. For instance, at the corner of a cube, the quadric cone passes through the three edges of the cube which meet there.t Thus the various conjectures which were made by some of the older investigators as to the value of $\Delta V$ at the corner of a cube are now seen to be all meaningless, unless we attach some special kind of definition to the symbol.

If the point $(x, y)$ is an ordinary cusp on the boundary, the second derivates certainly exist. For it is easy to see that $|C|$ is less than

$$
2 \gamma\left|\rho_{1}-\rho_{2}\right| \int_{h}^{u}(\phi / r) d r
$$

es on p. 351. But here $\lim _{r=0}\left(\phi / r^{3}\right)$ is finite, so that we may write $\phi \leqslant A r^{\text {th }}$ throughout the integral.

Hence $|C|<4 \gamma A\left|\rho_{1}-\rho_{2}\right|\left[a^{\frac{k}{2}}-h^{\natural}\right]$, and so $\lim _{h=0} C$ exists, and may be made

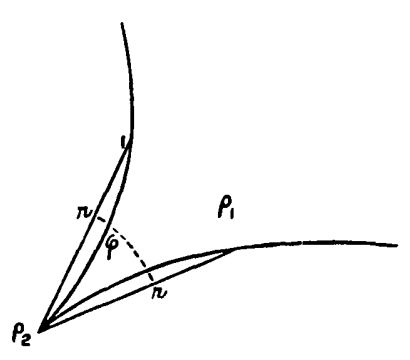
arbitrarily small by taking $a$ small enough.

* Trans. Roy. Soc. Edin., Vol. xxvı., 1872, p. 729 ; Electricity and Mayneti.mm, Vol. Ir., $\$ \$ 691-693$. The particular result required is (4) in $\$ 692$.

+ With these edges as axes of coordinates, the equation to the cone is $y z+z x+x y=0$. 'This equation is obtained by integrating out

$$
\iint\left[3\{(x \cos \phi+y \sin \phi) \sin \theta+z \cos \theta\}^{2}-\left(x^{2}+y^{2}+z^{2}\right)\right] \sin \theta d \theta d \phi
$$

over the appropriate solid angle, and equating the result to zero.

gBR. 2. roc. 3 . No. 904 . 
A simple example of a density which is continuous, but which gives a potential without second derivates at the origin, is

$$
\rho=\left(x^{2}-y^{2}\right) \sin \lambda / r^{2} \lambda,
$$

where $r^{2}=x^{2}+y^{2}$ and $\lambda=[\log (1 / r)]^{3}$; and, to make the density continuous, we must have $\rho=0$ at the origin. For then the integral $C$ is equal to

$$
2 \gamma \int_{0}^{2 \pi} \cos ^{2} 2 \theta d \theta \int_{h}^{a} \frac{\sin \lambda}{r \lambda} d r=4 \pi \gamma\left(\cos \lambda_{1}-\cos \lambda_{2}\right),
$$

where $\lambda_{1}=[\log (1 / h)]^{3}, \quad \lambda_{2}=[\log (1 / a)]^{3}$. Hence the limit $\lim _{h=0} C$ is quite indeterminate; and thus the second derivates $\partial^{2} V / \partial x^{2}, \partial^{2} V / \partial y^{2}$ cannot exist.

\section{A Lemma on the Limit of a Definite Integral.}

In the following sections $(\$ \S 4,5)$ we shall have to find the limiting values of certain definite integrals of the type

$$
\lim _{r=0} \int_{b}^{a / r} f(t, r) d t
$$

where $r$ approaches zero through positive values, and $a, b$ are fixed, $a$ being positive.

Suppose that

$$
\lim _{r=0} f(t, r)=g(t)
$$

then the truth of the equation

$$
\lim _{r=0} \int_{b}^{a / r} f(t, r) d t=\int_{b}^{\infty} g(t) d t
$$

depends (i.) on the convergence of the second integral, and (ii.) on some further condition. Without attempting to obtain the most general form of the second condition, the following will be found sufficient for the applications in view :-

It will be supposed that a number $N$ can be found, corresponding to any prescribed positive number $\epsilon$, such that

$$
\left|\int_{T}^{a / r} f(t, r) d t\right|<\epsilon,
$$

if $N<T<a / r$; this inequality holding for all values of $r$ less than $a / N$.

That some such condition is necessary is shown by the example

$$
f(t, r)=\frac{1}{1+t^{2}}-\frac{r}{1+r^{2} t^{2}},
$$


which gives $g(t)=1 /\left(1+t^{2}\right)$. But the integral

$$
\int_{0}^{1 / r} f(t, r) d t=\arctan (1 / r)-\frac{1}{4} \pi ;
$$

so that

$$
\lim _{r=0} \int_{0}^{1 / r} f(t, r) d t=\frac{1}{4} \pi,
$$

although

$$
\int_{0}^{\infty} g(t) d t=\frac{1}{2} \pi \text {. }
$$

To see that the conditions above are sufficient, we observe that when they are satisfied $N, N^{\prime}$ can be found so that

if $N \leqslant T<a / r$, and

$$
\left|\int_{T}^{a / r} f(t, r) d t\right|<\frac{1}{3} \epsilon
$$

$$
\left|\int_{\Gamma}^{\infty} g(t) d t\right|<\frac{1}{3} \epsilon
$$

if $N^{\prime} \leqslant T$.

Let $P$ denote the greater of $N, N^{\prime}$; then it is easily seen that.

$$
\left|\int_{P}^{a / r} f(t, r) d t-\int_{P}^{\infty} g(t) d t\right|<:_{3}^{2} \epsilon,
$$

provided that $r$ is less than $a / P$.

Since $P$ is now fixed, and since

$$
\lim _{r=0} f(t, r)=g(t),
$$

we can find $\delta$ so that

$$
|f(t, r)-g(t)|<\frac{1}{3} \epsilon /(P-b), \text { if } r<\delta,
$$

where $t$ is free to vary from $b$ to $P$. Then

$$
\begin{aligned}
\mid \int_{b}^{a / r} f(t, r) d t & -\int_{b}^{\infty} g(t) d t \mid \\
& \leqslant\left|\int_{b}^{P} f(t, r) d t-\int_{b}^{P} g(t) d t\right|+\left|\int_{P}^{a_{1} \tau} f(t, r) d t-\int_{P}^{\infty} g(t) d t\right|<\epsilon,
\end{aligned}
$$

provided that $r$ is less than both $a / P$ and $\delta$. Consequently

$$
\lim _{r=0} \int_{b}^{a / r} f(t, r) d t=\int_{b}^{\infty} g(t) d t .
$$

It is plain that exactly similar tests will justify the equation

$$
\lim _{r=0} \int_{-a / r}^{b} f(t, r) d t=\int_{-\infty}^{b} g(t) d t .
$$




\section{The First Derivates of the Logarithmic Potential of a Curve \\ (at a point on the curve).}

We shall assume that the curve has a tangent at all points with which we are concerned; further, that the curvature is finite and that the density $(\sigma)$ is continuous on the two sides of $O$, with a possible discontinuity at $O$.

Take the origin at the point $O$, at which the derivates are to be calculated, and let the axis of $x$ be the tangent at $O$, the axis of $y$ being the normal. Draw the two lines $x= \pm a$, which will cut out a small portion of the

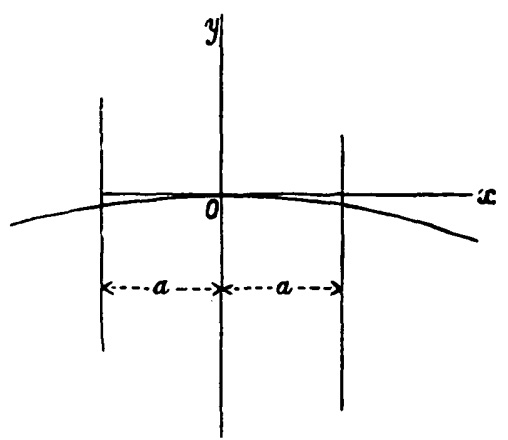
curve round $O$; then the potential of the remaining part of the curve is continuous and has continuous derivates of all orders, ${ }^{*}$ for points which are sufficiently close to $O$. Write, for the coordinates of points on the curve, $\eta=\xi \tan \psi$, so that $\psi$ is a function of $\hat{\xi}$ with the property that $\lim _{\xi=0}(\psi / \xi)$ is finite.

Then the potential at $(x, y)$ is

$$
-2 \gamma \int_{-a}^{+a} k \sigma \log R d \hat{\xi}
$$

where $R^{2}=(x-\xi)^{2}+(y-\eta)^{2}$, and $k$ is the secant of the angle between the tangent and the axis of $x$; in the same way the potential at $O$ is

$$
-\gamma \int_{-a}^{+a} k \sigma \log \left(\xi^{2}+\eta^{2}\right) d \xi
$$

Write $x=r l, y=r m$, where $l^{2}+m^{2}=1$; then, to find the derivate at $O$, in the direction of $l, m$, we have to examine

$$
-\frac{\gamma}{r} \int_{-a}^{+a} k \sigma \log \left(\frac{R^{2}}{\xi^{2}+\eta^{2}}\right) d \xi
$$

and see whether this has a limit when $r$ tends to zero. If we write $\xi=r t$, the integral to be considered becomes

$$
-\gamma \int_{-a \mid r}^{+a / r} k \sigma \log \left(\frac{\lambda^{2}}{t^{2} \sec ^{2} \psi}\right) d t
$$

* The proof of this statement offers no difficulty: the method of $\$ 1$ needs but little change to apply here. 
in which it must be remembered that $k, \sigma$, and $\psi$ are functions of $r t$, and

$$
\lambda^{2}=t^{2} \sec ^{2} \psi-2 t(l+m \tan \psi)+1 .
$$

The integral may be written in the form $A+B$, where

$$
\begin{aligned}
A= & -\gamma \int_{1}^{a / r} k \sigma\left[\log \left(\frac{\lambda^{2}}{t^{2} \sec ^{2} \psi}\right)+\frac{2}{t}(l \cos \psi+m \sin \psi) \cos \psi\right] d t \\
& -\gamma \int_{-a / r}^{-1} k \sigma\left[\log \left(\frac{\lambda^{2}}{t^{2} \sec ^{2} \psi}\right)+\frac{2}{t}(l \cos \psi+m \sin \psi) \cos \psi\right] d t \\
& -\gamma \int_{-1}^{+1} k \sigma \log \left(\frac{\lambda^{2}}{t^{2} \sec ^{2} \psi}\right) d t
\end{aligned}
$$

and

$$
\begin{aligned}
B= & 2 \gamma \int_{r}^{a} k \sigma(l \cos \psi+m \sin \psi) \cos \psi d \xi / \xi \\
& +2 \gamma \int_{-a}^{-r} k \sigma(l \cos \psi+m \sin \psi) \cos \psi d \xi \mid \xi .
\end{aligned}
$$

To the integral $A$ we can apply the lemma of $\$ 3$, and we find

$$
\begin{aligned}
\lim _{r=0} A= & -\gamma \sigma_{1}\left[\int_{0}^{1} \log \left(\frac{\lambda_{\rho}^{2}}{t^{2}}\right) d t+\int_{1}^{\infty}\left\{\log \left(\frac{\lambda_{0}^{2}}{t^{2}}\right)+\frac{2 l}{t}\right\} d t\right] \\
& -\gamma \sigma_{2}\left[\int_{-\infty}^{-1}\left\{\log \left(\frac{\lambda_{0}^{2}}{t^{2}}\right)+\frac{2 l}{t}\right\} d t+\int_{-1}^{0} \log \left(\frac{\lambda_{0}^{2}}{t^{2}}\right) d t\right],
\end{aligned}
$$

where $\sigma_{1}, \sigma_{2}$ are the limiting values of $\sigma$, on the two sides of $O$, and

$$
\lambda_{0}^{2}=t^{2}-2 l t+1 \text {. }
$$

To verify that the condition of the lemma is actually satisfied here, we observe that, if $h$ is between 0 and 1 ,

$$
\log \left(1-2 h \cos \theta+h^{2}\right)+2 h \cos \theta=-2\left(\frac{1}{2} h^{2} \cos 2 \theta+\frac{1}{3} h^{3} \cos 3 \theta+\ldots\right) ;
$$

so that

Hence

$$
\left|\log \left(1-2 h \cos \theta+h^{2}\right)+2 h \cos \theta\right|<h^{2} /(1-h)^{2} .
$$

$\left|\log \left(\frac{\lambda^{2}}{t^{2} \sec ^{2} \psi}\right)+\frac{2}{t}(l \cos \psi+m \sin \psi) \cos \psi\right|<1 /(t \sec \psi-1)^{2}<1 /(t-1)^{2}$, by taking $h=1 /(t \sec \psi)$; and consequently, if $N<T<a / r$, $\left|\gamma \int_{T}^{a / r} k \sigma\left[\log \left(\frac{\lambda^{2}}{t^{2} \sec ^{2} \psi}\right)+\frac{2}{t}(l \cos \psi+m \sin \psi) \cos \psi\right] d t\right|$

$$
<\int_{T}^{a / r} \frac{L d t}{(t-1)^{2}}<\frac{L}{N-1}
$$


where $L$ is the upper limit to $\gamma k \sigma$ in the small arc of the curve. It is now clear that the lemma may be applied to the first term in $A$; and a similar argument can be used for the second term in $A$; the third term requires no special discussion, since the range of the integral is fixed and includes no singularity of the integrand other than $t=0$ (which offers no difficulty).

After carrying out the integrations, it will be found that

$$
\begin{aligned}
\lim _{r=0} A= & -2 \gamma \sigma_{1}\left(1-l^{2}\right)^{\frac{1}{2}}\left[\frac{\pi}{2}+\arctan \frac{l}{\left(1-l^{2}\right)^{\frac{1}{2}}}\right] \\
& -2 \gamma \sigma_{2}\left(1-l^{2}\right)^{\frac{1}{2}}\left[\frac{\pi}{2}-\arctan \frac{l}{\left(1-l^{2}\right)^{3}}\right]-4 \gamma l\left(\sigma_{1}-\sigma_{2}\right) .
\end{aligned}
$$

As regards $B$, we note that

$$
\begin{aligned}
& \int_{r}^{u} k \sigma(l \cos \psi+m \sin \psi) \cos \psi d \xi \mid \xi \\
& \quad=\int_{r}^{a} k \sigma(m \cos \psi-l \sin \psi) \sin \psi d \xi\left|\xi+\int_{r}^{a} l(k-1) \sigma d \xi\right| \xi+\int_{r}^{a} l \sigma d \xi \mid \xi .
\end{aligned}
$$

In the first of these three integrals we have

$$
|m \cos \psi-l \sin \psi|<1,
$$

and $\lim _{\xi=0}(\sin \psi / \xi)$ is finite; so that the limit

$$
\lim _{r=0} \int_{r}^{a} k \sigma(m \cos \psi-l \sin \psi) \sin \psi d \xi \mid \xi
$$

exists, and may be made arbitrarily small by choice of $a$. In the second integral $\int_{r}^{a} l(k-1) \sigma d \xi|\xi| l \mid,<1$ and $\lim _{\xi=0}(k-1) / \xi=0$, in consequence of the finite curvature. Thus this integral has also a limit (as $r$ tends to zero) which may be made small by proper choice of $a$.

Thus the existence of the $\operatorname{limit} \lim _{r=0} B$ depends on that of

$$
\lim _{r=0}\left[\int_{r}^{a} l \sigma d \xi\left|\xi+\int_{-a}^{-r} l \sigma d \xi\right| \xi\right]=\lim _{r=0}\left[\int_{r}^{a} l\{\sigma(\xi)-\sigma(-\xi)\} d \xi \mid \xi\right] .
$$

Now, if, as we have assumed above, the two limits

$$
\lim _{\xi=0} \sigma(+\xi)=\sigma_{1}, \quad \lim _{\xi=0} \sigma(-\xi)=\sigma_{2}
$$

are different, it is plain that the last integral has no limit unless $l$ is zero. Thus we obtain the result : 
If $O$ is a point of discontinuity in $\sigma$, the general derivate does not exist, but the normal derivate does exist, and is given by

$-\pi \gamma\left(\sigma_{1}+\sigma_{2}\right)+$ terms which do not depend on the distribution near 0.

It may be noted that this derivate is calculated for the directions away from $O$; so that the discontinuity at $O$ in $\partial V / \partial y$ is $-2 \pi \gamma\left(\sigma_{1}+\sigma_{2}\right)$.

As a verification we may refer to Muxwell's result for a uniform rod [l.c., $\$ 692(1)]$; the derivate at $A$, in the direction $A O$, is given by*

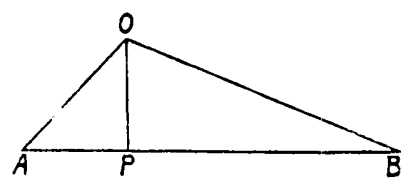

$$
-2 \gamma \sigma \lim _{O A=0} \frac{[A P \log O A+P B \log O B+O P(\angle A O B)]-A B \log A B}{O A},
$$

where $O P$ is perpendicular to $A B$.

In general this limit does not exist, but, if $O A$ is perpendicular to $A B$, so that $P$ is coincident with $A$, it gives the limit

$$
-2 \gamma \sigma \lim _{0, A=0}(\angle A O B)=-\pi \gamma \sigma,
$$

which agrees with our last two results, by taking $\sigma_{1}=\sigma, \sigma_{2}=0$.

For the existence of the general derivate it is, therefore, necessary that $\sigma$ should be continuous at $O$; but this condition is not sufticient, as may be seen from the example

$$
\begin{array}{ll}
\sigma=1+1 / \log (1 / \xi) & (\hat{\xi}>0), \\
\sigma=1 & (\xi \leqslant 0) .
\end{array}
$$

Here $\int_{r}^{a}[\sigma(+\xi)-\sigma(-\xi)] d \xi / \xi=\int_{r}^{a}[\log (1 / \xi)]^{-1} d \xi / \xi=\log \left[\frac{\log (1 / r)^{-}}{\log (1 / a)}\right]$,

which manifestly has no limit as $r$ tends to zero.

Thus we may conclude that:

The derirete (in any direction other than the normal) can only exist if the limit

$$
\lim _{r=0} \int_{r}^{a}[\sigma(\xi)-\sigma(-\xi)] d \hat{\xi}_{l}^{\prime} \xi
$$

exists, a condition which implies the continuity of $\sigma$. When this condition is satistied, the derivate is given by

$-2 \pi y \sigma_{0}\left(1-l^{2}\right)^{3}+$ terms. which !o not depend on the distributiun ncur $U$, where $\sigma_{0}$ is the bulue of $\sigma$ at $O$.

* In Maxwell's notation $A B \log R$ represents the value of the inteyral $\mid$ log $r a \xi$, inteyrated along the rod ; this is to be multiplied by $-2 \gamma \sigma$ to atgree with the form used here for the potential. 
It is easy to extend the results obtained here to cover the case when the curve consists of two distinct branches (each regular) meeting at $O$ in different directions. A short calculation shews that there is generally only one direction in which the derivate can exist, ${ }^{*}$ and even for this one a

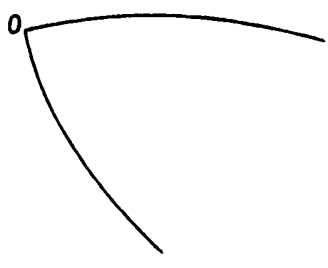
further condition (on $\sigma$ ) has to be satisfied.

For the existence of derivates in all directions, it is necessary that $\sigma$ should tend to zero at $O$ (on both branches), and that the integral $\int_{0} \sigma(\xi) d \xi / \xi$ should be convergent (for both branches separately). The case when $O$ is an ordinary cusp is also easy to discuss ; $\dagger$ the derivate in a direction perpendicular to the tangent always exists. For other directions the existence of the limit

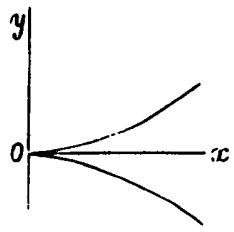

$$
\lim _{r=0} \int_{r}^{a}\left[\sigma_{1}(\xi)+\sigma_{2}(\xi)\right] d \xi / \xi
$$

is necessary.

\section{Components of Force (at a Point on the Curve) due to the Logarithmic Potential of a Curve.}

We investigated, in the last paragraph, the values of $\partial V / \partial x, \partial V / \partial y$ at $O$; but it does not follow, without special investigation, that these results give the components of force $\ddagger$ at $O$. We shall now examine, first, the limiting values of $\partial V / \partial x, \partial V / \partial y$ as $(x, y)$ moves up to $O$ along a regular arc which does not touch the curve at $O$; and, secondly, the values of the components of force at $O$.

We take the same axes and notation generally as in $\S 4$; then the force at $(x, y)$ in the direction $l, n$ is given by the integral

$$
2 \gamma \int_{-a}^{+a} \frac{k \sigma}{R^{2}}[l(\xi-x)+m(\eta-y)] d \xi,
$$

where $R^{2}=(\xi-x)^{2}+(\eta-y)^{2}$. Here, of course, this only represents the force due to the small arc of the curve round $O$; the force due to the remaining part of the curve is continuons within a sufficiently small area round the point $O$.

- In the case when $\sigma$ has the same limit at $O$ for both branches, this direction bisects the angle between the two tangents at $O$.

+ Of course, certain obvious changes must be made in the previous work, owing to the discontinuity in the curvature at $U$. Compare the work at the foot of p. 353 .

‡For the general proof that $\partial V / \partial x, \partial V / \partial y$ represent the components of force requires that $(x, y)$ shall not be a point of the curre. 
Write now $x=r f, y=r g$, where $f^{2}+g^{2}=1$; and, without loss of generality, $f, g$ may be supposed to remain constant as $r$ tends to zero; of course, it must be remembered that $g$ is not to be zero, though $f$ may be zero. Then, as before, put $\xi=r t, \eta=\xi \tan \psi$, and the limit to be considered becomes

$$
\lim _{r=0} 2 \gamma \int_{-a / r}^{+a / r} k \sigma \frac{t(l+m \tan \psi)-(l f+m g)}{t^{2} \sec ^{2} \psi-2 t(f+g \tan \psi)+1} d t,
$$

where now, of course, $k, \sigma, \psi$ are functions of the product $r t$.

Now this integral may be put in the form $A+B$, where

$$
\begin{aligned}
A & =2 \gamma \int_{-a / r}^{-1} k \sigma\left[\frac{t(l+m \tan \psi)}{t^{2} \sec ^{2} \psi-2 t(f+g \tan \psi)+1}-\frac{1}{t}(l \cos \psi+m \sin \psi) \cos \psi\right] d t \\
& +2 \gamma \int_{-1}^{+1} k \sigma \frac{t(l+m \tan \psi)}{t^{2} \sec ^{2} \psi-2 t(f+g \tan \psi)+1} d t \\
& +2 \gamma \int_{+1}^{+a / r} k \sigma\left[\frac{t(l+m \tan \psi)}{t^{2} \sec ^{2} \psi-2 t(f+g \tan \psi)+1}-\frac{1}{t}(l \cos \psi+m \sin \psi) \cos \psi\right] d t \\
& -2 \gamma \int_{-a / r}^{+a / r} k \sigma \frac{(l f+m g) d t}{t^{2} \sec ^{2} \psi-2 t(f+g \tan \psi)+1}
\end{aligned}
$$

and

$$
\begin{aligned}
B= & 2 \gamma \int_{-a}^{-r} k \sigma(l \cos \psi+m \sin \psi) \cos \psi d \xi / \xi \\
& +2 \gamma \int_{r}^{a} k \sigma(l \cos \psi+m \sin \psi) \cos \psi d \xi / \xi .
\end{aligned}
$$

It will be seen that this $B$ is the same as the one used in the last paragraph; so that no fresh investigation of the limit of $B$ will be required. Of course, $A$ is not now the same as before. To evaluate the limit of $A$, we apply the lemma of $\S 3$, and we consider first

$$
\left|\int_{T}^{a / r} \frac{(l f+m g) d t}{t^{2} \sec ^{2} \psi-2 t(f+g \tan \psi)+1}\right| .
$$

Here $|l f+m g| \leqslant 1,|f+g \tan \psi| \leqslant \sec \psi$; so that this expression is less than

$$
\int_{T}^{a / r} \frac{d t}{(t \sec \psi-1)^{2}}<\int_{T}^{a / r} \frac{d t}{(t-1)^{2}}<\frac{1}{T-1} ;
$$

thus, if $N=1+1 / \epsilon$, and $a / r>T>N$, the test of the lemma is satisfied, provided that $|k \sigma|$ remains less than a fixed number on the small arc of the curve. 
A similar argument applies to the case

and consequently

$$
\left|\int_{-a / r}^{-r} \frac{(l f+m g) d t}{t^{2} \sec ^{2} \psi-2 t(f+g \tan \psi)+1}\right|
$$

$$
\begin{aligned}
\lim _{r=0} 2 \gamma \int_{-a r}^{+a r} k \sigma & \frac{(l f+m g) d t}{t^{2} \sec ^{2} \psi-2 t(f+g \tan \psi)+1} \\
& =2 \gamma \sigma_{1} \int_{0}^{+\infty} \frac{(l f+m g) d t}{t^{2}-2 f t+1}+2 \gamma \sigma_{2} \int_{-\infty}^{0} \frac{(l f+m g) d t}{t^{2}-2 f t+1} \\
& =2 \gamma \sigma_{1} \frac{l f+m g}{\left(1-f^{2}\right)^{\frac{l}{2}}}\left(\frac{\pi}{2}+a\right)+2 \gamma \sigma_{2} \frac{l f+m g}{\left(1-f^{2}\right)^{3}}\left(\frac{\pi}{2}-\alpha\right),
\end{aligned}
$$

where

$$
a=\arctan \left[f /\left(1-f^{2}\right)^{k}\right] \text {. }
$$

Take next

$$
\begin{aligned}
\left|\int_{t}^{a r}\left[\frac{(l+m \tan \psi) t}{t^{2} \sec ^{2} \psi-2 t(f+g \tan \psi)+1}-\frac{1}{t}(l \cos \psi+m \sin \psi) \cos \psi\right] d t\right| \\
=\left|\int_{r}^{a / r} \frac{(l \cos \psi+m \sin \psi)[2 t(j \cos \psi+g \sin \psi)-\cos \psi]}{t\left[t^{2} \sec ^{2} \psi-2 t(f+g \tan \psi)+1\right]} d t\right|
\end{aligned}
$$

here: $l \cos \psi+m \sin \psi: \leqslant 1, i f \cos \psi+g \sin \psi: \leqslant 1$; so that the expression is less than

$$
\int_{T}^{a r} \frac{2 t+1}{t(t \sec \psi-1)^{2}}<\int_{T}^{u r} \frac{3}{(t-1)^{2}}<\frac{3}{T-1},
$$

If $T>1$. Thus, just as above, the test of the lemma is satisfied; so that $\lim _{r=1,} 2 \gamma \int_{1}^{m r} k \sigma\left[\frac{(l+m \tan \psi) t}{t^{2} \sec ^{2} \psi-2 t(f+g \tan \psi)+1}-\frac{1}{t}(l \cos \psi+m \sin \psi) \cos \psi\right] d t$

Similariy,

$$
=2 \gamma \sigma_{1} \int_{1}^{\infty}\left(\frac{l t}{t^{2}-2 f t+1}-\frac{l}{t}\right) d t \text {. }
$$

$$
\begin{aligned}
& \lim _{n=6} 2 \gamma \int_{-a / r}^{-1} k \sigma\left[\frac{(l+m \tan \psi) t}{\left.t^{2} \sec ^{2} \psi-2 t(t)+g \tan \psi\right)+1}-\frac{1}{t}(l \cos \psi+m \sin \psi) \cos \psi\right] d t \\
& =2 \gamma \sigma_{2} \int_{-x}^{-1}\left(\frac{l t}{t^{2}-2 f t+1}-\frac{l}{t}\right) d t
\end{aligned}
$$

and, without any special investigation,

$$
\begin{aligned}
\lim _{,-u} 2 \gamma \int_{-1}^{+1} k \sigma \frac{\left(l+m \cdot \tan \psi_{\sigma}\right) t}{t^{2} \sec ^{2} \psi-2 t(f+g \tan \psi)+1} d t & \\
& =2 \gamma \sigma_{1} \int_{-\nu}^{1} \frac{l t}{t^{2}-2 f t+1} d t+2 \gamma \sigma_{2} \int_{-1}^{n} \frac{l t}{t^{2}-2 f t+1} d t .
\end{aligned}
$$


The sum of these four integrals is found to be

$$
2 \gamma \sigma_{1} \frac{l f}{\left(1-f^{2}\right)^{\frac{1}{2}}}\left(\frac{\pi}{2}+\alpha\right)+2 \gamma \sigma_{2} \frac{l f}{\left(1-f^{2}\right)^{\frac{1}{3}}}\left(\frac{\pi}{2}-\alpha\right) .
$$

Hence $\lim _{r=0} A=-2 \gamma \sigma_{1} \frac{m g}{\left(1-f^{2}\right)^{2}}\left(\frac{\pi}{2}+\alpha\right)-2 \gamma \sigma_{2} \frac{m g}{\left(1-f^{2}\right)^{2}}\left(\frac{\pi}{2}-\alpha\right)$,

where it should be observed that the value of $g /\left(1-f^{2}\right)^{\frac{1}{1}}$ is either +1 or -1 , according to the sign of $g$.

We saw above that when $\sigma_{1}$ and $\sigma_{2}$ are unequal the limit of $B$ could only exist if $l=0$; if we write $l=0$ and $n l=1$, we see that-

In case $\sigma$ is discontinuous at $O$, the limit of the normal force as $(x, y)$ approaches $O$ is only equal to the normal derivate of the potential at $O$, if $(x, y)$ approaches $O$ along the normal.* In general, the value of the limit depends on the direction of the path followed by $(x, y)$.

The limit of the tangential force cannot exist.

As a verification, consider the case of a uniform straight rod; the result of Maxwell's, already used (p. 359), shows that

$$
\frac{\partial V}{\partial x}=-2 \gamma \sigma \log (O A / O B), \quad \frac{\partial V}{\partial y}=-2 \gamma \sigma(\angle A O B),
$$

where now $O$ is the point $(x, y)$. The origin is taken at the end $A$ of the rod, with the axis of $x$ along the rod. It is clear that $\partial V / \partial x$ has no limit when $O$ approaches $A$, while $\partial V / \partial y$ approaches the limit

$$
-2 \gamma \sigma\left[\frac{\pi}{2}+\arctan (f / g)\right],
$$

assuming that $y$ (and therefore $g$ ) is positive. This agrees with the limit calculated above, if we write $\sigma_{1}=\sigma, \sigma_{2}=0$.

Turning now to the case when $\sigma$ is continuous at $O$, so that

$$
\sigma_{1}=\sigma_{2}=\sigma_{0}, \text { say, }
$$

it is clear that

$$
\lim _{r=0} A=\mp 2 \pi \gamma \sigma_{0} m \text {, }
$$

* Because $f$ is then zero; so that $\lim _{r=0} A=\mp \pi \gamma\left(\sigma_{1}+\sigma_{2}\right)$, the sign $\mp$ being opposite to the sign of $g$.

In general, $f=\sin a$ and the value is found to be $\mp\left[\pi \gamma\left(\sigma_{1}+\sigma_{\Omega}\right)+2 a \gamma\left(\sigma_{1}-\sigma_{8}\right)\right]$. 
where the ambiguous sign is opposite to the sign of $g$. Comparing this with the result at the foot of p. 359, we can therefore state:-

The force (in a direction not parallel to the normal at 0 ) has a limiting value only if the limit

$$
\lim _{r=0} \int_{r}^{a}[\sigma(+\xi)-\sigma(-\dot{\xi})] d \xi \mid \xi
$$

exists, a condition which implies the continuity of $\sigma$. When this condition is satisfied, the limit of the force is

$\mp 2 \pi \gamma \sigma_{0} v \imath+$ terms which do not depend on the distribution near $O$, which is the same as the derivate at 0 , in the same direction

The discussion of modifications of these results, consequent on geometrical singularities of the curve at $O$, may be carried out exactly as in the last paragraph.

Finally, to calculate the components of force at $O$, we must consider the limiting value of

$$
\int_{r}^{a} k \sigma \frac{l \xi+m \eta}{\xi^{2}+\eta^{2}} d \xi+\int_{-a}^{-s} k \sigma \frac{l \xi+m \eta}{\xi^{2}+\eta^{2}} d \xi,
$$

when $r$ and $s$ tend to zero independently.

If, as before, we write $\eta=\xi \tan \psi$, we have to evaluate

$$
\begin{aligned}
\lim _{r, s=0}\left[\int_{r}^{a} k \sigma(l \cos \psi+m \sin \psi)\right. & \cos \psi d \xi / \xi \\
& \left.+\int_{-a}^{-a} k \sigma(l \cos \psi+m \sin \psi) \cos \psi d \xi / \xi\right] .
\end{aligned}
$$

Proceeding exactly as before (p. 358), we see that this is essentially the same as

$$
\lim _{r, s=0}\left[\int_{r}^{a} l \sigma d \xi\left|\xi+\int_{-a}^{-s} l \sigma d \xi\right| \xi\right] .
$$

Now, unless $\sigma$ tends to zero at $O$, this limit is not determinate when $l$ is different from zero. Thus we may state the result:

The normal force at $O$ is determinate, and is independent of the distribution near $O$. But the tangential force at $O$ is generally indeterminate even when $\sigma$ is continuous, unless $\sigma_{0}=0$.

We may call the value obtained by writing $s=r$ the principal value of the tangential force. It is then clear that the conditions for the existence of this principal value are exactly the same as for the existence of the general derivate of the potential at $O$. 
6. A Comparison of the foregoing Results with those of Previous Writers.

The only writer who has explicitly discussed these problems for the logarithmic potential is Harnack in his Theorie des logarithmischen Potentiales (Leipzig, 1887). However I shall refer to some authors who have obtained results for the corresponding problems in the Newtonian potential theory, because their conclusions generally apply to the logarithmic potential, if suitable modifications are made.

It should be observed, once for all, that all these writers make the preliminary assumption that the density is completely continuous; and consequently their conclusions cannot be compared with such of the foregoing results as relate to discontinuities in the density.

It will be convenient for the purposes of comparison to examine the surface distribution and line distribution separately; and to modify the conditions found previously, by introducing the hypothesis of continuity at the beginning (compare $\$ 7$ below).

(I.) Surface Distributions.-The existence of the second derivate $\partial^{2} V / \partial x^{2}$ was proved ( $\$ 2$ above) to depend on the limit

$$
\lim _{h=0} C=\lim _{h=0} \int_{0}^{2 \pi} \cos 2 \theta d \theta \int_{h}^{a}(\rho / r) d r .
$$

Now, if $\rho$ is continuous and $\lim _{r=0} \rho=\rho_{0}$, we have

$$
\int_{0}^{2 \pi} \cos 2 \theta d \theta \int_{h}^{a}\left(\rho_{0} / r\right) d r=\rho_{0} \log (a / h) \int_{0}^{2 \pi} \cos 2 \theta d \theta=0 ;
$$

so that the limit above is the same as

$$
\lim _{h=0} \int_{0}^{2 \pi} \cos 2 \theta d \theta \int_{h}^{\prime \prime}\left[\left(\rho-\rho_{0}\right) / r\right] d r=\lim _{h=0} C^{\prime}
$$

(I. a) Hölder's Condition (Inaugural Dissertation,* 'Tübingen, 1882).The condition is that

$$
\left|\rho-\rho_{0}\right|<G r^{g},
$$

where $G, g$ are positive constants, and $r$ is less than some fixed value, which we may take to be greater than $a$. If this holds,

$$
\begin{aligned}
\left|\int_{0}^{2 \pi} \cos 2 \theta d \theta \int_{r_{1}}^{r_{2}}\left[\left(\rho-\rho_{0}\right) / r\right] d r\right| & <2 \pi \int_{r_{1}}^{r_{2}}\left|\rho-\rho_{0}\right| r^{-1} d r<2 \pi G \int_{r_{1}}^{r_{2}} r^{g-1} d r \\
& <2 \pi(G / g)\left(r_{2}^{g}-r_{1}^{g}\right) .
\end{aligned}
$$

* This dissertation has unfortunately been inaccessible, but all subsequent writers agree in quoting the same form for the condition given by Hölder. 
Thus the limit of $C^{\prime}$ exists and can be made as small as we please by diminishing $a$; it is easily seen, further, that the condition is also satisfied when $h$ is negative, so that the derivate is not merely one-sided.

Harnack (l.c., p. 24, footnote) has remarked that Hölder's method for the Newtonian potential can be modified to apply to the logarithmic potential; and C. Neumann* has given an alternative discussion of Hölder's results which can also be modified, although the method of $\$ 7$ would seem to be preferable.

(I.b) Harnack's Condition (Theorie \&c., Kap. 1, §6). - It is here supposed that $\rho$ has finite and integrable first derivates- $a$ condition analogous to the one used by Gauss in discussing the Newtonian potential. Then, since

$$
\frac{\cos 2 \theta}{r^{2}}=-\frac{\partial}{\partial \xi}\left(\frac{\xi-x}{r^{2}}\right)
$$

the limit to be examined may be replaced by

$$
\lim _{h=0} \iint\left(\rho-\rho_{0}\right) \frac{\partial}{\partial \xi}\left(\frac{\xi-x}{r^{2}}\right) d \xi d \eta
$$

where the integral is extended over the ring between two circles of radii $h, a$ whose common centre is $(x, y)$.

This integral may be integrated by parts, and it becomes then

$$
\left[\int_{0}^{2 \pi}\left(\rho-\rho_{0}\right) \cos ^{2} \theta d \theta\right]_{r=h}^{r=a}-\iint \frac{\cos \theta}{r} \frac{\partial \rho}{\partial \xi} d \xi d \eta
$$

The integrals round the circles are together less, in absolute value, than $4 \pi \omega$, if $\omega$ is the greatest value of $\left|\rho-\rho_{0}\right|$ on either circle; and, since $\rho$ is continuous, $\omega$ can be made as small as may be required by diminishing $a$. The surface integral is less, $t$ in absolute value, than $2 \pi a M$, where $M$ is the greatest value of $|\partial \rho / \partial \xi|$ in the ring. Thus

$$
\iint\left(\rho-\rho_{0}\right) \frac{\partial}{\partial \xi}\left(\frac{\xi-x}{r^{2}}\right) d \xi d \eta
$$

- Lcipzig Berichte, Bd. xurr., 1890, p. 327.

+ This is almost obvious; reference may be made, if necessary, to the methods of $\$ 1$. 
may be made arbitrarily small by diminishing $a$; therefore the limit

$$
\lim _{h=0} \iint\left(\rho-\rho_{0}\right) \frac{\partial}{\partial \xi}\left(\frac{\xi-x}{r^{2}}\right) d \xi d \eta
$$

must exist, and consequently $\partial^{2} V / \partial x^{2}$ also exists.

(I.c) Morera's Condition. *-It is here assumed that the limit

$$
\lim _{h=0} \int_{h}^{a} \frac{\left(\rho-\rho_{0}\right)}{r} d r
$$

exists for all values of $\theta$; if this condition is satisfied, it is plain that the limit

$$
\lim _{h=0} \int_{0}^{2 \pi} \cos 2 \theta d \theta \int_{h}^{a}\left[\left(\rho-\rho_{0}\right) / r\right] d r
$$

exists, and the condition of $\$ 2$ is therefore satisfied.

(II.) Line Distributions.-In this connection, the previous results appear to relate entirely to the problem of $\S 5$, the calculation of the derivates at a point of the curve (as in $\$ 4$ ) not having been undertaken. In many cases, too, the point $(x, y)$ is supposed to approach the curve along the normal; as was proved above $(\$ 5)$, this limitation of the mode of approach does affect the iorce, if there is a discontinuity in the density. But in the older work the density is invariably assumed to be continuous, and consequently the limitation does not really make the results less general.

(II. a) Hölder's Condition (l.c.).-This is $\left|\sigma-\sigma_{0}\right|<G|\xi|^{g}$, where $G$ and $g$ are positive constants, and $|\xi|$ is less than some fixed value. When this holds the condition of $\$ 5$ is easily seen to be satisfied. For then

$$
\left|\int_{r}^{r^{2}}[\sigma(+\xi)-\sigma(-\xi)] d \xi\right| \xi \mid<2 G \int_{r_{1}}^{r_{3}} \xi^{g-1} d \xi<2 G r_{2}^{g} / g ;
$$

so that

$$
\lim _{r=0} \int_{r}^{a}[\sigma(+\xi)-\sigma(-\xi)] d \xi \mid \xi
$$

exists and may be made arbitrarily small by choice of $a$.

- G. Morera, Rend. del R. Ist. Lomb., Ser. 2, T. xx., 1887, p. 302, and Il Nuovo Cimento, Ser. 3, T. xxur., 1887, p. 240. Reference may also be made to G. A. Maggi, Rend. del R. Ist. Lomb., Ser. 2, T. xxir., 1889, p. 647 ; Il Nuovo Cimento, Ser. 3, T. xxvi., 1890, p. 21 ; T. xxxilr., 1893, p. 113. 
(II.b) Harnack's Condition (Theorie, \&c., Kap. 1, $\$ 9$ ). - It is assumed that $\sigma$ has a finite and integrable first derivate, say $\sigma^{\prime}(\xi)$; and then

$$
\lim _{\xi=0}[\sigma(+\xi)-\sigma(-\xi)] / \xi=2 \sigma^{\prime}(0) ;
$$

so that the limit $\quad \lim _{r=0} \int_{r}^{a}[\sigma(+\xi)-\sigma(-\xi)] d \xi / \xi$

certainly exists.

(II.c) Morera's Condition.*-It is assumed that the limits

$$
\lim _{r=0} \int_{r}^{a}\left|\left(\sigma-\sigma_{0}\right)\right| \xi \mid d \xi \text { and } \quad \lim _{r=0} \int_{-a}^{-r}\left|\left(\sigma-\sigma_{0}\right)\right| \xi \mid d \xi
$$

both exist. If this is so, it follows at once that the limit

$$
\lim _{r=0} \int_{r}^{a}|\sigma(+\xi)-\sigma(-\xi)| d \xi \mid \xi
$$

exists ; and consequently that the condition of $\$ 5$ is satisfied.

It is manifest that of the conditions enumerated Morera's are the nearest to those obtained in $\S \S 2,5$ above. However, they do not include all cases in which derivates exist; thus, with a surface distribution such that $\rho_{0}=0$ and $\rho=1 / \lambda$, where $\lambda=[\log (1 / r)]^{3}$, we have

$$
\int_{0}^{2 \pi} \cos 2 \theta d \theta \int_{h}^{a}(\rho / r) d r=0 ;
$$

so that the condition of $\S 2$ is satisfied, and the potential has second derivates. But

$$
\int_{h}^{a} \frac{\left|\rho-\rho_{0}\right|}{r} d r=2[\log (1 / h)]^{3}-2[\log (1 / a)]^{3} ;
$$

so that Morera's condition (I. $c$ ) would exclude this case.

Similarly, a line distribution such that

$$
\sigma_{0}=0, \quad \sigma=[\log (1 /|\xi|)]^{-1}
$$

would satisfy the condition of $\$ 5$, but not Morera's condition (II. $c$ ).

7. Simplified Investigations in case the Density is Continuous.

If $\rho$ is completely continuous, the work of $\S 2$ can be shortened considerably by the following metbod. Let $U$ denote the logarithmic potential of the circular area when it carries a uniform density $\rho_{0}$ (the

* G. Morera, Rend. del K. Ist. Lomb., Ser. 2, T. xx., 1887, p. 543 ; Il Nuovo Cimento, Ser. 3, T. xxir., 1888, p. 1. Compare G. A. Maggi, Rend. del R. Ist. Lomb., Ser. 2, T. xxrv., 1891, pp. 8i, 22C, 960 ; Il Ninovo Cimento, Ser. 3, T. xxxur., 1893, p. 249, T. xxxrv., 1894, pp. $22,81$. 
value of $\rho$ at the centre)* ; it is then obvious that

$$
\frac{\lambda^{2} V}{\partial x^{2}}=\frac{\partial^{2} U}{\partial x^{2}}+\lim _{h=0} \iint \frac{2 \gamma}{h}\left(\rho-\rho_{0}\right)\left(\frac{\xi-x-h}{r^{\prime 2}}-\frac{\xi-x}{r^{2}}\right) d \xi d \eta,
$$

and a short calculation shows that +

Thus

$$
\begin{gathered}
U=-2 \pi \gamma \rho_{0}\left[a^{2} \log a+\frac{1}{2}\left(r^{2}-a^{2}\right)\right] . \\
\frac{\partial^{2} V}{\partial x^{2}}=-2 \pi \gamma \rho_{0}+\lim _{h=11} I,
\end{gathered}
$$

if $I$ denotes the integral written above.

For brevity let $c$ denote $|h|$, and let $\omega$ denote the maximum of $\left|\rho-\rho_{0}\right|$ within the circle of radius $a$; let a circle of radius $2 c$ be drawn, with centre $(x, y)$, and let $I_{1}, I_{2}$ represent the parts of $I$ which arise from the area within the smaller circle, and from the ring between the circles. Then, by a method exactly like the methods used in $\$ 1$, we can prove that:

$$
\left|I_{1}\right|<\frac{2 \gamma \omega}{c} \iint\left(\frac{1}{r}+\frac{1}{r^{\prime}}\right) d \hat{\xi} d \eta<12 \pi \gamma \omega .
$$

To deal with $I_{2}$, we observe that

$$
\begin{aligned}
\frac{\dot{\xi}-x-h}{r^{\prime 2}}-\frac{\dot{\xi}-x}{r^{2}} & =\frac{r \cos \theta-h}{r^{2}-2 r h \cos \theta+h^{2}}-\frac{\cos \theta}{r} \\
& =\frac{h \cos 2 \theta}{r^{2}}+\frac{h^{2}(r \cos 3 \theta-h \cos 2 \theta)}{r^{2}\left(r^{2}-2 r h \cos \theta+h^{2}\right)} .
\end{aligned}
$$

Thus

$$
I_{2}-2 \gamma \int_{0}^{2 \pi} \cos 2 \theta d \theta \int_{2 c}^{a}\left[\left(\rho-\rho_{0}\right) / r\right] d r=K
$$

where

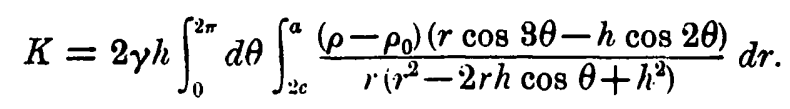

Hence

$$
|K|<4 \pi \gamma c \omega \int_{2 c}^{\infty} \frac{r+c}{(r-c)^{3}} d r
$$

or

$$
|\hbar|<4 \pi \gamma \omega \int_{1}^{\infty} \frac{t+2}{t^{3}} d t \quad \text { (putting } r=c+c t \text { ), }
$$

that is

$$
|K|<8 \pi \gamma \omega \text {. }
$$

It is now clear that $\partial^{2} V / \partial x^{2}+2 \pi \gamma \rho_{0}$ differs from

$$
\lim _{h=0} 2 \gamma \int_{0}^{2 \pi} \cos 2 \theta d \theta \int_{2 c}^{a}\left[\left(\rho-\rho_{0}\right) / r\right] d r
$$

* In fact, here the function $f(\theta)$ used in $\$ 2$ is the constant $\rho_{0}$.

+ This does nut appesr to be given by Maxwell (l.c.), but is easily deduced from his resultm.

+ It may be remarked that $I_{1}$ corresponds to $A+B$ of $\$ 2$, and $I_{2}$ to $C$.

SER. 2. voL. 3. no. 905 . 
by less than $\left|I_{1}\right|+|K|$; and therefore the difference is less than $20 \pi \gamma \omega$. But $\omega$ can be made arbitrarily small, by diminishing $a$, so that the existence of $\partial^{2} V / \partial x^{2}$ depends only on the existence of the limit

$$
\lim _{c=0} \int_{0}^{2 \pi} \cos 2 \theta d \theta \int_{c}^{a}\left[\left(\rho-\rho_{0}\right) / r\right] d r,
$$

which is the result of $\$ 2$ as modified for continuous density on p. 365 .

If $\sigma$ is continuous, it is similarly possible to simplify the work of $\$ \$ 4,5$ by comparing the potential of the actual curve with that of a short portion of the tangent at $O$, carrying a uniform density $\sigma_{0}$; but the simplification is not so great as to make it worth while to put down the details here.

In conclusion, it may be added that Petrini's investigations on the Newtonian potential of a volume density can be simplified in a manner similar to that described in this paragraph, if the density is known to be continuous at all points within a small sphere enclosing the point at which the second derivates are to be calculated. 